\title{
Mon patient comprend-il ce que je m'apprête à faire? Bref survol de la loi sur le consentement éclairé
}
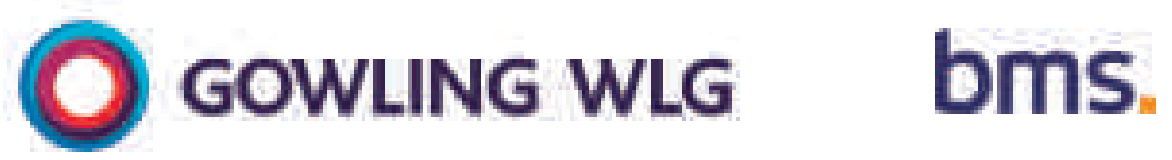

Cet article a été préparé et rédigé par l'équipe juridique de la Healthcare Professionals Insurance Alliance du $B M S$ Group chez Gowling WLG (Canada) LLP (Gowlings). Les membres de l'ACO qui participent au programme d'assurance responsabilité civile et professionnelle ont droit à 30 minutes de services pro bono et inclusifs de Gowlings en cas de poursuite pour responsabilité professionnelle. Gowlings est l'un des cabinets juridiques les plus importants et les mieux cotés au Canada dans les domaines de la défense médicale et de la responsabilité professionnelle.

\section{"Si j’avais su que cela pouvait m'arriver, je n'aurais jamais consenti à l'opération. "}

Les avocats des professionnels de la santé ont entendu ce commentaire d'innombrables fois. Dans les affaires médico-légales, les patients allèguent souvent qu'ils n'ont pas donné leur consentement éclairé à une intervention qui a fini par leur causer des lésions ou y a contribué.

En tant que fournisseurs de soins de santé, les optométristes ont l'obligation juridique d'obtenir le consentement de leur patient avant chaque traitement. Si vous n'obtenez pas le consentement au traitement, vous vous exposez à une poursuite civile et/ou à une convocation devant l'organisme de réglementation de votre province.

Pour être valide, le consentement au traitement doit être un traitement « éclairé ». Le patient doit avoir reçu une explication raisonnable de la nature de l'investigation ou du traitement proposé et du résultat à prévoir, ainsi que des risques importants auxquels il s'expose et des solutions de rechange possibles. L'information fournie doit être suffisante pour permettre au patient de prendre une décision éclairée.

En plus des principes généraux qui sous-tendent la doctrine du consentement éclairé, l'organisme de réglementation de chaque province a ses propres politiques et/ou directives de pratique en matière de consentement éclairé. En outre, certaines provinces ont imposé l'obligation statutaire d'obtenir le consentement éclairé (p. ex., la Loi sur le consentement aux soins de santé en Ontario et la Loi sur le consentement aux soins de santé et l'admission dans les services de santé en Colombie-Britannique). Enfin, en plus des directives sur la pratique ou des responsabilités statutaires, la common law oblige à obtenir le consentement éclairé au traitement. L'information présentée ici est donc de nature plutôt générale. Il est vivement recommandé de communiquer avec l'organisme de réglementation de votre province si vous avez des questions précises au sujet des exigences applicables du secteur de compétence où vous exercez.

Au Canada, pour être valide, le consentement du patient doit satisfaire aux critères suivants :

1. Le patient doit avoir la capacité de consentir au traitement.

2. Le fournisseur de soins doit faire une divulgation appropriée de l'information au patient.

3. L’autorisation doit viser l'intervention spécifique à pratiquer.

4. Le patient devrait avoir la possibilité de :

a. poser des questions,

b. recevoir des réponses claires pour lui.

5. L'autorisation obtenue doit être libre de toute influence indue et de toute coercition.

6. L'autorisation obtenue doit être libre de toute présentation erronée de l'information importante. 


\section{Le patient doit avoir la capacité de consentir au traitement}

Un consentement n'est valide que si la personne qui le donne a la capacité de le faire. La question de la compétence légale se pose typiquement lorsque le patient n'a pas 18 ans ou peut souffrir d'une maladie mentale quelconque. Cependant, ces facteurs ne devraient pas déterminer à eux seuls la compétence (c. à d. une personne qui n’a pas 18 ans ou qui a un déficit cognitif peut donner un consentement valide au traitement).

Pour évaluer la capacité, vous devez avoir confiance que la personne consentant au traitement peut comprendre la nature et les conséquences de la discussion sur le consentement. En cas de doute, demandez le consentement du père ou de la mère, du tuteur ou du décideur substitut. S'il est possible que le patient ne comprenne pas la nature et les conséquences de la discussion sur le consentement à cause d'un problème de langue, faites venir quelqu'un pour traduire.

\section{Le fournisseur de soins doit faire une divulgation appropriée de l'information au patient}

Votre patient doit comprendre la nature du traitement et la raison pour laquelle vous le lui proposez. Vous devez informer le patient des risques associés au traitement. Il y a une question typique au sujet de la mesure dans laquelle le patient doit connaître les risques. Au Canada, vous êtes tenu de mettre le patient au courant des risques inhérents, importants et spécifiques. Les risques inhérents sont les plus communs. Les risques importants sont ceux qui sont moins communs, mais graves s'ils surviennent. Les risques importants peuvent différer d'un patient à l'autre, si bien que vous devez tenir compte de l'état de santé et de la condition de votre patient dans la détermination des risques qui sont importants. Enfin, les risques spécifiques comprennent ceux qui sont possibles pour le patient particulier.

Au Canada, pour déterminer si le patient a donné son consentement éclairé, il faut voir si une personne raisonnable moyenne, se trouvant dans la même situation que le patient, aurait consenti au traitement si elle avait connu les risques inhérents, importants et spécifiques.

En plus de la divulgation qui précède, il faut informer le patient des conséquences possibles du traitement sur son mode de vie, et des considérations économiques associées à la réception ou au refus du traitement proposé. Il faut informer le patient des autres traitements possibles et des risques et avantages de chacun. Enfin, il faut l'informer des risques que comporte un refus de traitement.

\section{L'autorisation doit viser l'intervention spécifique à pratiquer}

Le consentement que donne le patient doit viser le traitement/l'intervention spécifique que vous proposez ou recommandez.

Il n'est pas nécessaire d'obtenir le consentement du patient à chaque étape du plan de traitement. Cependant, le formulaire de consentement général, comme celui que le patient doit typiquement signer au moment de son admission dans une clinique ou un bureau privé, ne suffit pas. Si la méthode de traitement que vous proposez pour un patient consiste en une série de traitements échelonnés dans le temps, il n'est pas nécessaire d'obtenir un consentement pour chaque étape du traitement. Cependant, il faut discuter de l'ensemble du traitement avec le patient.

Si d'autres personnes participent au traitement (p. ex., un étudiant, un assistant optométrique, etc.), alors vous devez bien informer le patient du fait que d'autres personnes interviendront au traitement et obtenir le consentement du patient à leur intervention.

\section{Le patient devrait avoir la possibilité de poser des questions et de recevoir des réponses claires pour lui}

La discussion concernant le consentement ne doit pas être monologue. Idéalement, vous devriez avoir une conversation où le patient pourra poser des questions et vous pourrez donner l'information nécessaire en réponse à ces questions.

\section{L'autorisation obtenue doit être libre de toute influence indue et de toute coercition}

Vous devez bien vérifier que votre patient ne se sent pas contraint ni obligé d'aller de l'avant avec le traitement proposé. Non seulement vous devez vérifier que le patient ne subit pas de pressions d'un tiers, mais encore vous devez veiller, en tant que fournisseur des soins, à ne pas préconiser le plan de traitement ou l'intervention de telle manière que le patient estimerait ne pas avoir d'autre choix que de l'accepter. 


\section{L'autorisation obtenue doit être libre de toute présentation erronée de l'information importante}

Vous êtes libre de donner au patient votre opinion sur la meilleure ligne de conduite à suivre, mais vous devez être aussi objectif que possible dans la présentation de l'information au patient. Vous devez donner une information exacte et impartiale sur tous les traitements possibles.

\section{DOCUMENTATION DE LA DISCUSSION SUR LE CONSENTEMENT}

Il est essentiel de documenter la discussion que vous avez avec votre patient au sujet du consentement. Vous avez peut être une pratique type au sujet de la discussion que vous avez avec vos patients avant d'entreprendre un traitement, mais cela ne remplace pas la nécessité de documenter votre discussion. Idéalement, vous devriez discuter du plan de traitement proposé avec le patient, documenter la discussion, puis faire signer le plan de traitement par le patient. Cependant, au minimum, vous devez documenter le fait que vous avez parlé au patient, lui avez expliqué le plan de traitement ou l'intervention et en avez exposé les risques et les avantages, et l'avez informé des autres solutions possibles. Vous devez aussi noter les questions que le patient avait à poser en précisant si le patient a donné son consentement.

Dans une affaire médico-légale, où le consentement éclairé fait partie du contentieux, le patient peut soutenir que vous ne lui avez pas donné toute l'information nécessaire pour prendre une décision éclairée. Si vous avez documenté votre discussion, cela devrait faciliter la preuve que vous avez effectivement donné toute l'information nécessaire. L'absence de documentation concernant une discussion éclairée augmente les chances que le tribunal ou l'organisme de réglementation arrive à la conclusion que vous n'avez pas donné l'information nécessaire au patient.

En conclusion, en intégrant une politique détaillée de consentement éclairé dans les procédures normales de votre pratique, vous pouvez diminuer votre responsabilité et donner à vos patients l'information à laquelle la loi et l'éthique leur donnent droit.

Prière de noter que ce commentaire n'est pas un avis juridique, et qu'il ne faut pas l'utiliser comme tel. Si vous avez des questions au sujet du consentement éclairé dans votre pratique, communiquez avec l'Association canadienne des optométristes (ACO) et/ou l'organisme de réglementation de votre province. 